\title{
Análisis del Impacto Ambiental de la Recuperación de Metanol en la Producción de Biodiesel usando el Algoritmo de Reducción de Desechos WAR
}

\author{
Alfredo A. Marticorena ${ }^{1}$, Beatriz A. Mandagarán ${ }^{2}$ y Enrique A. Campanella ${ }^{2,3^{\star}}$ \\ (1) Universidad Nacional del Litoral, Facultad de Ingeniería Química, Santiago del Estero 2654, \\ 3000 Santa Fe-Argentina \\ (2) INTEC (Universidad Nacional del Litoral - CONICET), Güemes 3450, 3000 Santa Fe-Argentina \\ (3) Universidad Nacional del Litoral, Facultad de Ingeniería y Ciencias Hídricas, Ruta \\ Nacional No168 Km 472.4, Santa Fe-Argentina (e-mail: tquique@santafe-conicet.gov.ar)
}

* autor a quien debe ser dirigida la correspondencia

\section{Resumen}

En este trabajo se realiza un análisis del impacto ambiental de la recuperación de metanol en el proceso convencional de producción de Biodiesel. El biodiesel se presenta como una alternativa para disminuir el impacto ambiental de los combustibles tradicionales. Sin embargo, existen distintos procesos de producción de biodiesel y todos ellos tienen un mayor o menor impacto en el ambiente. La metodología consiste en simular los procesos, aplicarles el algoritmo de reducción de desechos WAR y comparar los resultados. Los resultados de este estudio se comparan también con otros similares de la literatura. El análisis indica que recuperar metanol transforma al proceso convencional de producción de biodiesel en un proceso amigable para el medio ambiente.

Palabras clave: biodiesel, algoritmo WAR, impacto ambiental, simulación de procesos, recuperación de metanol

\section{Environmental Impact Analysis of Methanol Recovery in Biodiesel Production using the Waste Reduction Algorithm WAR}

\begin{abstract}
The environmental impact of recovering methanol in the traditional process of biodiesel production is presented. Biodiesel represents an alternative for diminishing impact on the environment. However, there are several biodiesel production processes and all of them have different impact on the environment. The methodology consists of simulating the process, apply the WAR algorithm for waste reduction and compare the results. Comparison is also done with literature data for similar cases. The analysis shows that methanol recovery transforms the conventional process of biodiesel production into an environmental friendly process.
\end{abstract}

Keywords: biodiesel, WAR algorithm, environmental impact, process simulation, methanol recovery 


\section{INTRODUCCIÓN}

Biodiesel es un término genérico que refiere a ésteres de ácidos grasos obtenidos por transesterificación de aceites vegetales con un alcohol (generalmente metanol). El biodiesel es por lo tanto renovable y se presenta como sustituto del diesel obtenido del petróleo con ventajas desde el punto de vista ambiental.

La transesterificación puede ser catalizada por catalizadores homogéneos ácidos y básicos (Gerpen, 2005), catalizadores heterogéneos (Vasudevan y Briggs, 2008), catalizadores enzimáticos (Ranganathan et al., 2008), en condiciones supercríticas (Vasudevan y Briggs, 2008). Por otra parte existen varias posibilidades para el aceite vegetal: comestible, no comestible, de alga o aceite vegetal usado (Vasudevan y Briggs, 2008). Para la etapa de refinación del biodiesel se presentan distintas alternativas dependiendo de la materia prima y del tipo de catalizador usado (Jordan y Gutsche, 2001; Karaosmanoğlu et al., 1996). La producción ha sido analizada desde el punto de vista económico (Zhang et al., 2003b; Haas et al., 2006). También el análisis del ciclo de vida ha sido aplicado para comparar biodiesel con diesel (Sheehan et al., 1996), comparar la producción utilizando catalizador básico con la producción enzimática (Harding et al., 2008) y comparar producción convencional (catalizador básico) con producción supercrítica (Kiwjaroun et al., 2009).

Para analizar el impacto de los procesos sobre el ambiente, el método más desarrollado es el análisis de ciclo de vida (LCA) (Burgess y Brennan, 2001). Otra de las herramientas usadas para la generación de indicadores ambientales para procesos químicos es el algoritmo de reducción de desechos (WAR) desarrollado por la agencia de medio ambiente de Estados Unidos (EPA). El algoritmo se basa en la determinación del potencial de impacto ambiental (PEI) que es una cantidad conceptual que representa el impacto que las emisiones de materia y energía producirán en el ambiente (Young y Cabezas, 1999; Young et al., 2000; Cardona et al., 2004).

En este trabajo se comparan el impacto ambiental de dos alternativas de diseño (casos 1 y 2) para una planta de biodiesel. El caso 1 genera efluentes que tienen metanol (componente tóxico). El caso 2 incorpora el tratamiento y reutilización de los efluentes tóxicos incrementando el consumo energético. Ambas alternativas tienen ventajas y desventajas ambientales que necesitan cuantificarse.

\section{METODOLOGÍA}

Para evaluar el impacto ambiental se siguió la metodología de trabajo llamada WAR (Waste Reduction) (Young y Cabezas, 1999), que arroja valores de impacto ambiental para las categorías detalladas a continuación, acompañadas de sus siglas en inglés: Potencial intoxicación humana por ingestión (HTPI); Potencial intoxicación humana por exposición dérmica o inhalación (HTPE); Potencial toxicidad terrestre (TTP); Potencial toxicidad acuática (ATP); Potencial calentamiento global (GWP); Potencial destrucción de ozono (ODP); Potencial formación de smog fotoquímico (PCOP); Potencial de acidificación (AP). Cada una de estas categorías componen, finalmente, un índice total. Como en otros estudios de este tipo, ocurre que diferentes objetivos ambientales presentan conflictos. Es decir que al mejorar un aspecto se estarían deteriorando otros. Por lo tanto, estos estudios apuntan en definitiva a revelar la incidencia y comportamiento de cada una de las categorías ambientales involucradas frente a las distintas alternativas de diseño.

\section{Descripción equilibrios}

Para realizar las simulaciones el primer paso es definir los componentes de la mezcla y el modelo termodinámico a utilizar. Para la simulación se considera que el sistema está formado por triglicérido, metanol, metiléster, glicerol e hidróxido de sodio. Para el triglicérido y su éster se eligieron la trioleína y el metiloleato como su éster metílico o biodiesel. La elección se basó en su disponibilidad en la base de datos de UniSim Design R380, aunque se hicieron algunas simulaciones con aceite de soja siguiendo el procedimiento discutido por Mandagarán y Campanella (2008). Teniendo en cuenta la presencia de compuestos polares en el proceso (metanol, glicerol) se eligió utilizar el modelo termidinámico NRTL (Renon y Prausnitz, 1968) como paquete de propiedades para la simulación de los procesos. Se ajustaron los parámetros binarios del modelo NRTL de manera que los sistemas binarios y ternarios representaran adecuadamente datos experimentales. Un ejemplo del ajuste 
conseguido se presenta en la Fig. 1. La figura muestra una comparación de los cálculos con Simulis ${ }^{\circledR}$ Thermodynamics para el equilibrio líquido-líquido del sistema metanol-glicerol-éster con datos de Negi et al. (2006) y de Andreatta et al. (2008). Se realizaron también comparaciones con los datos de Chiu et al. (2005) y de Zhou y Boocock (2006).
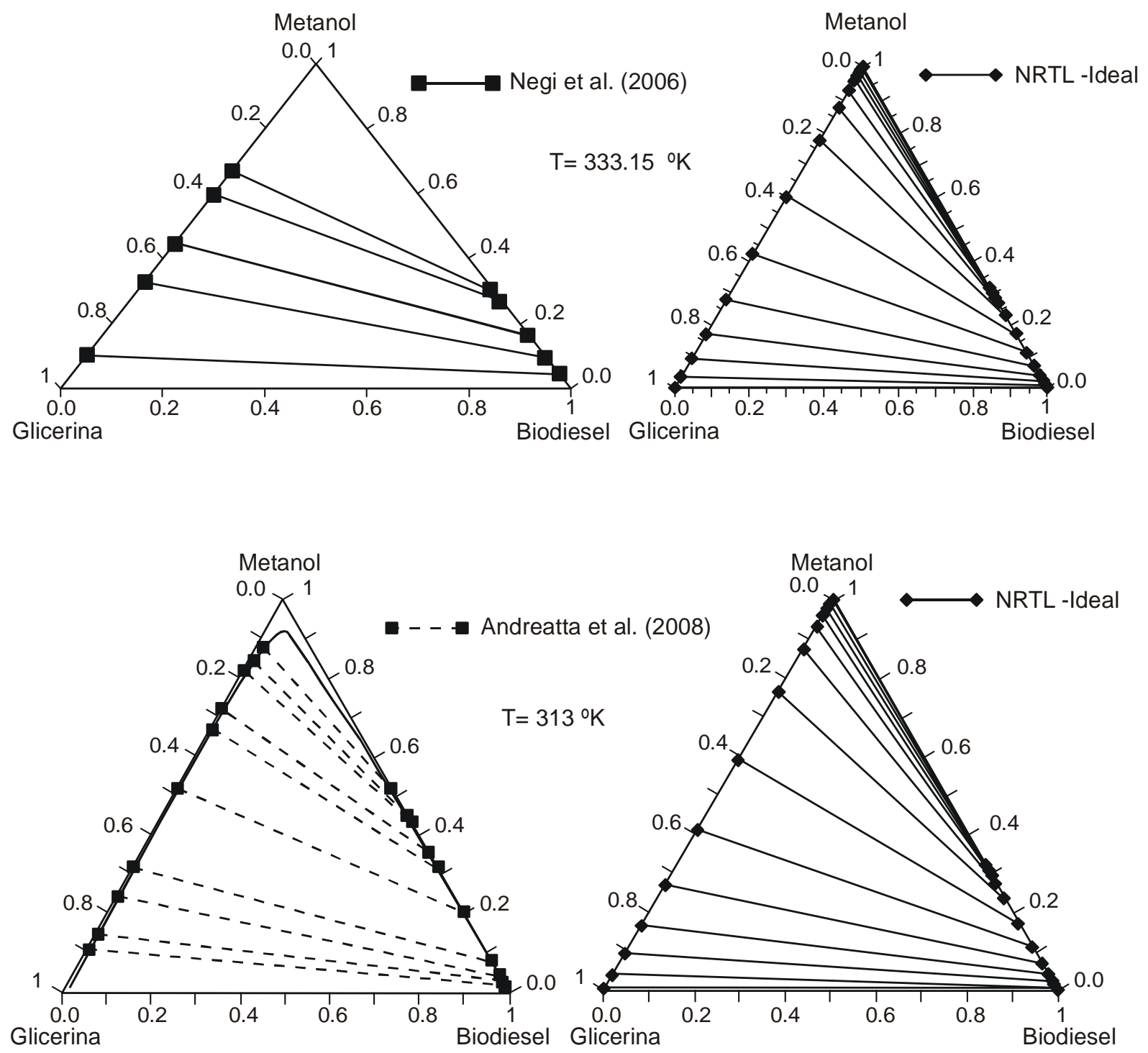

Fig. 1: Equilibrio líquido-líquido para el sistema biodiesel-glicerina-metanol a distintas temperaturas

Tabla 1: Características de los equipos de los procesos

\begin{tabular}{|l|c|c|}
\hline Equipo & Caso 1 & Caso 2 \\
\hline Reactor R1 & $96 \%$ conv. & $96 \%$ conv. \\
\hline Reactor R2 & $96 \%$ conv. & $96 \%$ conv. \\
\hline Torre T1 & $5(4), 2^{\mathrm{a}}$ & $5(3), 2^{\mathrm{a}}$ \\
\hline Torre T2 $^{\mathrm{a}}$ & $5(-),-^{\mathrm{a}}$ & $5(-),-^{\mathrm{a}}$ \\
\hline Torre T3 $^{\mathrm{a}}$ & $5(4), 6^{\mathrm{a}}$ & $5(4), 6^{\mathrm{a}}$ \\
\hline Torre T4 & $5(3), 2^{\mathrm{a}}$ & $5(3), 2^{\mathrm{a}}$ \\
\hline Torre T5 & - & $7(5,7), 6.47^{\mathrm{a}}$ \\
\hline $\begin{array}{l}\text { a. Número de platos (plato de alimentación), } \\
\text { relación de reflujo }\end{array}$ \\
\hline
\end{tabular}




\section{Descripción simulación}

Los diagramas de flujo de las alternativas estudiadas fueron simulados con UniSim Design R380, las especificaciones de los equipos fueron definidas previamente utilizando herramientas tradicionales de diseño y recomendaciones de Zhang et al. (2003a; 2003b). La Tabla 1 presenta las principales especificaciones de los procesos simulados.

La Fig. 2 muestra el diagrama de flujo del proceso para el caso 1: en una primera etapa los reactivos (aceite de soja y metanol) junto con el catalizador (hidróxido de sodio o de potasio) ingresan en una batería de dos reactores de transesterificación (R1 y R2) en los que se alcanza una conversión global mayor a 99.8\%. El producto de reacción está compuesto por dos fases: una rica en biodiesel (metil éster de aceite de soja) y otra rica en glicerina; además dicha corriente contiene cantidades importantes de metanol sin reaccionar ya que en el sistema de reacción se encuentra en exceso. Más del 90\% del metanol se recupera en la columna de destilación T1 y se recircula al proceso. La corriente que sale por fondo de T1 ingresa en una columna de lavado con agua, T2, en la que se lleva a cabo la separación de fases, a la vez que se transfiere a la fase acuosa buena parte del metanol y catalizador presente en la fase orgánica. Por tope se obtiene biodiesel ligeramente impuro que ingresa a la columna de destilación T3 en la que se purifica hasta cumplir con las especificaciones del producto. La corriente de fondo de T2 está compuesta por glicerina, agua de lavado, catalizador y gran parte del metanol sin reaccionar. Luego de una etapa de neutralización, dicha corriente alimenta una torre de destilación, T4, en la que se purifica la glicerina.

El diseño presenta dos aspectos que lo hacen amigable con el medio ambiente: la recuperación de metanol en T1 y la integración de calor para precalentar la alimentación de aceite y enfriar la salida de fondo de T1. Sin embargo, la generación de efluentes es un problema importante teniendo en cuenta que se trata de un proceso de capacidad media-alta (2000 kg/h de biodiesel) y que las corrientes de desecho Tope T3 y Tope T4 contienen metanol en distintas proporciones (50 kg/h de efluentes líquidos aproximadamente).

En la etapa de neutralización se agrega ácido fosfórico, se genera entonces un producto sólido (sal) que no se tiene en cuenta en este estudio ambiental. La glicerina obtenida como subproducto tiene varios usos aunque debido al aumento de producción mundial de biodiesel, el precio de la glicerina está bajando y las alternativas de utilización constituyen un tema de estudio aparte.

El caso 2 (Fig. 3) agrega al de la Fig. 2 una columna de destilación alimentada a distintas alturas por las corrientes Tope T3 y Tope T4, que separa metanol como destilado y una fase rica en agua por el fondo. Ambas se recirculan al proceso, la primera se utiliza como reactivo y la segunda para la etapa de lavado.

Una vez que se tienen los resultados de las simulaciones de interés (caudal y composición de las corrientes de entrada y salida al sistema, consumo energético), se calcula el impacto ambiental según la metodología WAR utilizando el software, WAR Versión 1.0.15.

\section{RESULTADOS Y DISCUSIÓN}

Los principales resultados del estudio de presentan en las Tablas 2 y 3 y en la Fig. 4 . La Tabla 2 resume la simulación del proceso de producción de biodiesel de la Fig. 2 (caso 1). La cantidad de metanol recuperado es del orden del que se suministra por alimentación. El calor recuperado en el intercambio entre corriente de alimentación al reactor (R1) con la corriente de salida de la columna de destilación (T1) es de $1.30 \mathrm{E}+05 \mathrm{~kJ} / \mathrm{h}$. Este valor es aproximadamente un 10\% del total de la energía puesta en juego en el proceso. La Tabla 3 resume la simulación del proceso de producción de biodiesel de la Fig. 3 (caso 2). Este esquema recupera metanol adicional de la corriente que abandona el tope de la columna T3. Por un lado existe una reducción en la alimentación de metanol de $253.41 \mathrm{~kg} / \mathrm{h}$ en la corriente AR1 (alimentación a bomba P1) a $237.65 \mathrm{~kg} / \mathrm{h}$ en el nuevo esquema. Por otro lado existe un incremento del requerimiento de energía de $3.79 \mathrm{E}+06 \mathrm{~kJ} / \mathrm{h}$ a $4.04 \mathrm{E}+06 \mathrm{~kJ} / \mathrm{h}$, proveniente de la tarea térmica de la torre T3. 
Finalmente la Fig. 4 presenta los resultados de impacto ambiental obtenidos del programa WAR. Dos tendencias son evidentes. Por un lado, los índices GWP y AP, globales y relacionados con el uso de energía son mayores en el caso 2. Por otro lado, los índices HTPI, HTPE, TTP, PCOP se anulan en el caso 2 al eliminar los efluentes que contaminan con metanol. Los índices ODP (no se produce emisión gaseosa que afecte el ozono) y ATP (no hay cambios en la corriente residual de glicerina) no cambian en los casos 1 y 2.

Si bien los resultados de este trabajo parecen limitados, los mismos cobran otra dimensión si se los pone en el contexto de trabajos similares publicados en la literatura y mencionados en la Introducción.

La herramienta más recomendada para comparar impacto ambiental es un análisis de ciclo de vida (LCA) (Burgess y Brennan, 2001). Como ya fue citado, se puede encontrar comparación entre el proceso convencional (catalizador básico) y el proceso enzimático (Harding et al., 2008). En este trabajo se utiliza el software SimaPro (2006) y el sistema motivo de análisis es lo que se denomina "de cuna a tumba" para la producción de biodiesel incluyendo todas las materias primas y demandas de energía involucradas en la producción agrícola y el procesamiento del biodiesel. Utilizando esa información podemos, por un lado, comparar no solo las modificaciones al proceso convencional con el proceso convencional en sí, sino también incluir los procesos enzimáticos y supercríticos, y por otro lado utilizar WAR que solo evalúa los índices de impacto ambiental del proceso de producción de biodiesel, no preocupándose por lo que sucede antes (cultivo de soja) ni después (distribución del biodiesel).

Si se incorporan las conclusiones de los trabajos que realizan estudios de LCA para el proceso convencional versus el proceso supercrítico (Kiwjaroun et al., 2009) y para el proceso convencional versus el proceso enzimático (Harding et al., 2008) a los resultados obtenidos en este trabajo, se puede amplificar la comparación del impacto ambiental de la recuperación de metanol propuesta para el proceso convencional. Dado que el metanol es el causante de la mayoría del impacto ambiental, el proceso enzimático es más amigable que el proceso convencional y éste es más amigable que el proceso supercrítico. La alternativa de mejorar la performance ambiental del proceso de producción de biodiesel reemplazando metanol por etanol no se incluye en a discusión. La modificación propuesta, recuperación de metanol, convierte al proceso convencional en un proceso con impacto ambiental del orden del proceso enzimático.

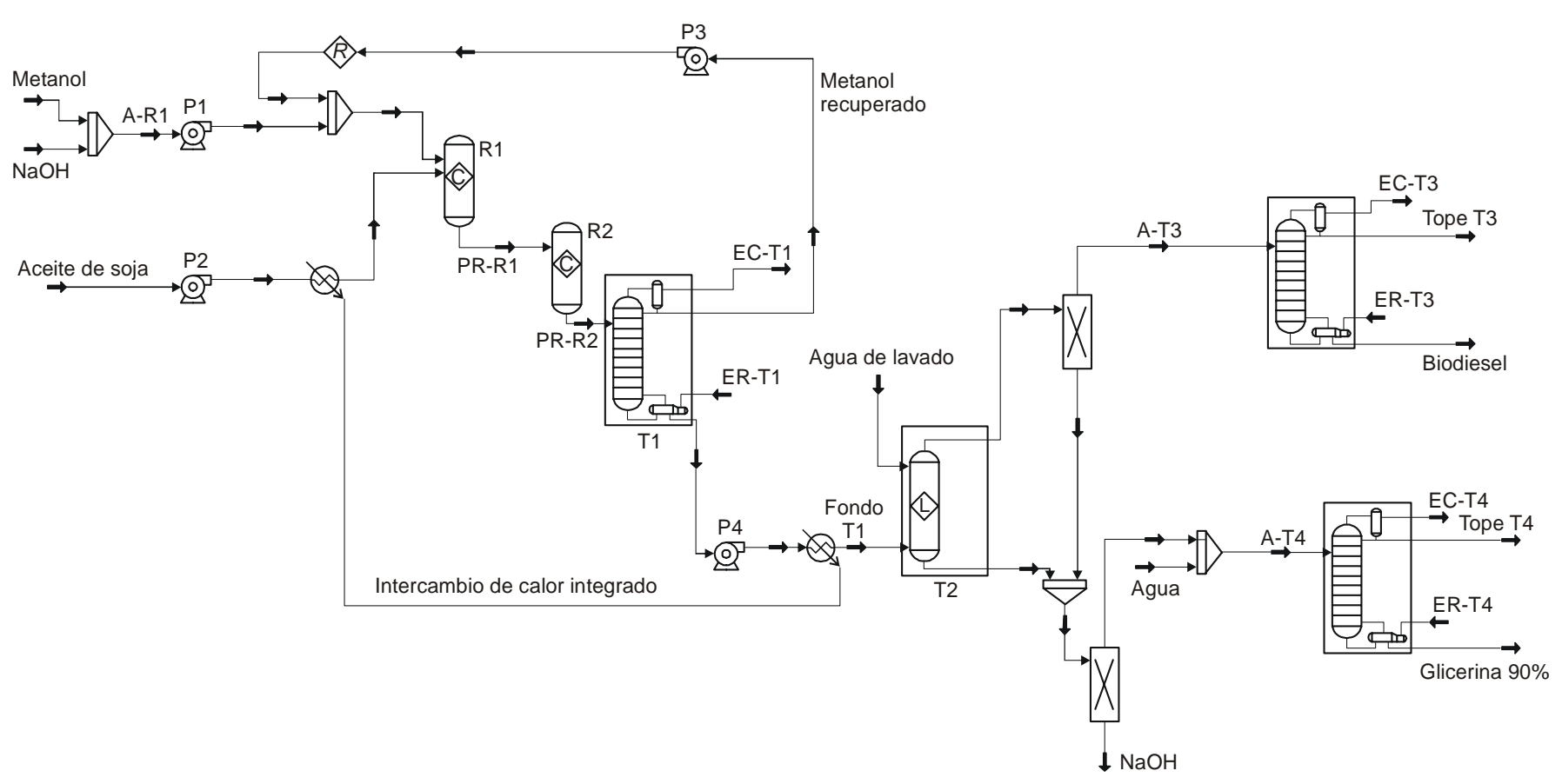

Fig. 2: Diagrama de flujo del proceso de producción de biodiesel a partir de aceite de soja. Caso 1 
Tabla 2: Principales resultados de la simulación del caso 1

\begin{tabular}{|c|c|c|c|c|c|c|c|c|c|c|c|c|c|}
\hline Corriente & $\begin{array}{l}\text { Aceite } \\
\text { de soja }\end{array}$ & AR-1 & PR-2 & \begin{tabular}{c|} 
Metanol \\
recuperado
\end{tabular} & Fondo T1 & $\begin{array}{c}\text { Agua de } \\
\text { lavado }\end{array}$ & A-T3 & Biodiesel & Tope T3 & A-T4 & $\begin{array}{c}\text { Glicerina } \\
(90 \%)\end{array}$ & Tope T4 & Metanol \\
\hline Frac.Vap. & 0,000 & 0,000 & 0,000 & 0,000 & 0,000 & 0,000 & 0,000 & 0,000 & 0,000 & \begin{tabular}{|l|}
0,000 \\
\end{tabular} & 0,000 & 0,000 & 0,000 \\
\hline Temp. ${ }^{\circ} \mathrm{C}$ & 25,00 & 32,94 & 60,00 & 28,20 & 67,05 & 27,00 & 63,92 & 321,09 & 38,65 & 67,45 & 103,38 & 46,03 & 25,00 \\
\hline Pres. kPa & 100 & 100 & 400 & 20 & 100 & 100 & 100 & 30 & 20 & 100 & 30 & 20 & 100 \\
\hline Flujo Mas. kg/h & 2000,00 & 253,41 & 2456,39 & 203,00 & 2253,39 & 70,00 & 2012,23 & 2008,40 & 3,83 & 300,17 & 231,82 & 68,36 & 233,41 \\
\hline Frac.Mas.(Biodiesel) & 0,000 & 0,000 & 0,817 & 0,000 & 0,890 & 0,000 & 0,997 & 0,998 & 0,001 & 0,002 & 0,000 & 0,009 & 0,000 \\
\hline Frac.Mas.(Ac.Soja) & 1,000 & 0,000 & 0,001 & 0,000 & 0,001 & 0,000 & 0,002 & 0,002 & 0,000 & 0,000 & 0,000 & 0,000 & 0,000 \\
\hline Frac.Mas.(Glicerina) & 0,000 & 0,000 & 0,085 & 0,000 & 0,093 & 0,000 & 0,000 & 0,000 & 0,000 & 0,695 & 0,900 & 0,000 & 0,000 \\
\hline Frac.Mas.(Metanol) & 0,000 & 0,921 & 0,089 & 1,000 & 0,007 & 0,000 & 0,001 & 0,000 & 0,453 & 0,047 & 0,000 & 0,205 & 1,000 \\
\hline Frac.Mas.(Agua) & 0,000 & 0,000 & 0,000 & 0,000 & 0,000 & 1,000 & 0,001 & 0,000 & 0,547 & 0,256 & 0,100 & 0,786 & 0,000 \\
\hline Frac.Mas.(NaOH) & 0,000 & 0,079 & 0,008 & 0,000 & 0,009 & 0,000 & 0,000 & 0,000 & 0,000 & 0,000 & 0,000 & 0,000 & 0,000 \\
\hline
\end{tabular}

\begin{tabular}{|c|c|c|c|c|c|c|c|c|c|c|c|c|c|}
\hline \multirow{2}{*}{ 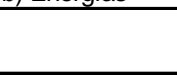 } & \multicolumn{4}{|c|}{ Hervidores } & \multicolumn{4}{|c|}{ Condensadores } & \multicolumn{5}{|c|}{ Bombas } \\
\hline & ER-T1 & ER-T3 & ER-T4 & SUMA & EC-T1 & EC-T3 & EC-T4 & SUMA & $\mathrm{P} 1$ & $\mathrm{P} 2$ & P3 & P4 & SUMA \\
\hline Flujo Calor kJ/h & $8,62 \mathrm{E}+05$ & $1,26 \mathrm{E}+06$ & $4,56 \mathrm{E}+05$ & $2,58 \mathrm{E}+06$ & $7,21 \mathrm{E}+05$ & $5,05 \mathrm{E}+04$ & $4,38 \mathrm{E}+05$ & $1,21 \mathrm{E}+06$ & & & & & \\
\hline \begin{tabular}{|l|} 
Potencia kJ/h \\
\end{tabular} & & & & & & & & & 128,72 & 274,43 & 131,42 & 229,66 & 764,23 \\
\hline
\end{tabular}

\begin{tabular}{|l|l|}
\hline Energía total & $3,79 \mathrm{E}+06$ \\
\hline
\end{tabular}

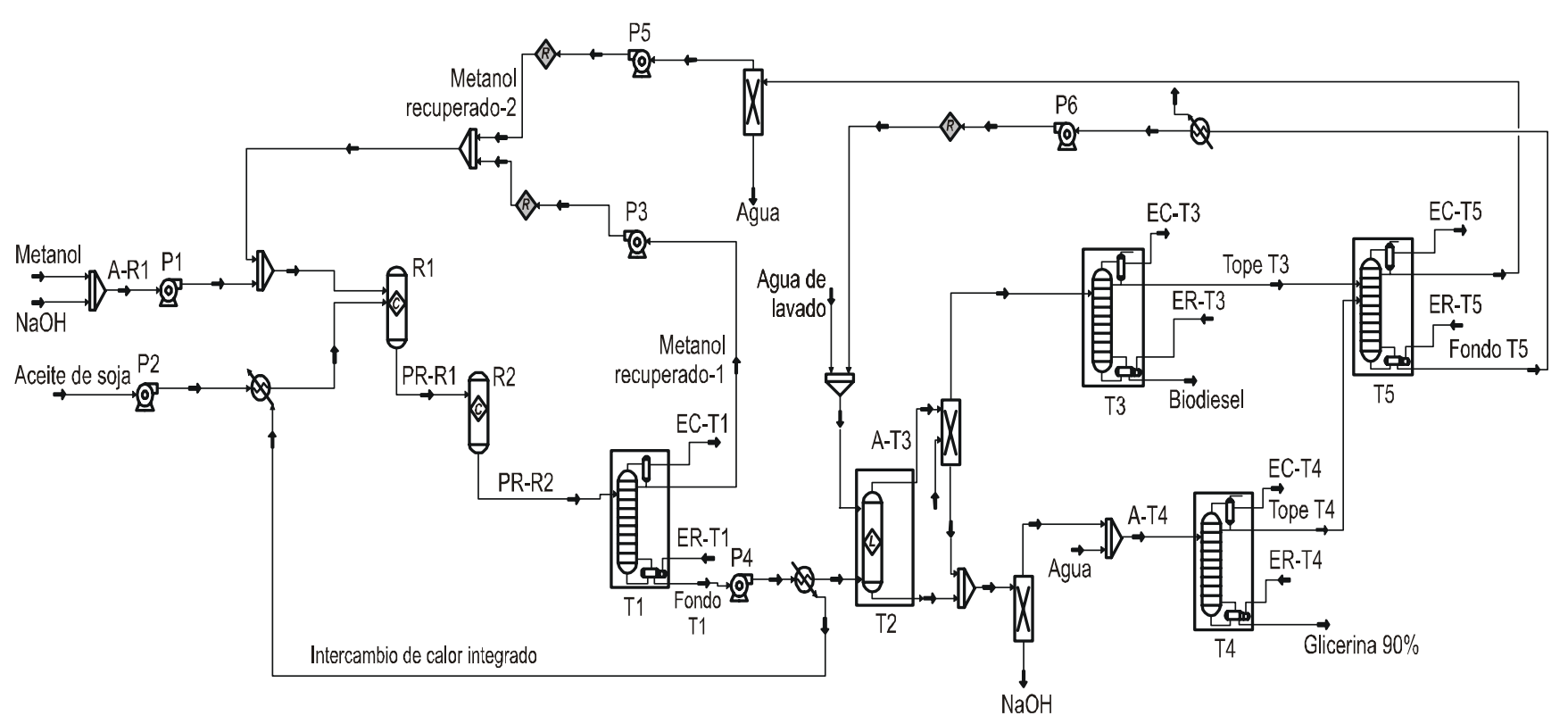

Fig. 3: Diagrama de flujo del proceso de producción de biodiesel a partir de aceite de soja. Caso 2

Tabla 3: Principales resultados de la simulación del caso 2

\begin{tabular}{|c|c|c|c|c|c|c|c|c|c|c|c|c|c|c|c|}
\hline Corriente & $\begin{array}{c}\text { Aceite } \\
\text { de soja }\end{array}$ & AR-1 & PR-2 & $\begin{array}{l}\text { Metanol } \\
\text { recup.-1 }\end{array}$ & Fondo T1 & $\begin{array}{l}\text { Agua de } \\
\text { lavado }\end{array}$ & $\mathrm{A}-\mathrm{T} 3$ & Biodiesel & Tope T3 & A-T4 & \begin{tabular}{|c|} 
Glicerina \\
$(90 \%)$
\end{tabular} & \begin{tabular}{|c|} 
Tope \\
T4
\end{tabular} & Metanol & \begin{tabular}{|l|} 
Metanol \\
recup.-2
\end{tabular} & \begin{tabular}{|c|} 
Fondo \\
T5
\end{tabular} \\
\hline Frac.Vap. & 0,000 & 0,000 & 0,000 & 0,000 & 0,000 & 0,000 & 0,000 & 0,000 & 0,000 & 0,000 & 0,000 & 0,000 & 0,000 & 0,000 & 0,000 \\
\hline Temp. ${ }^{\circ} \mathrm{C}$ & 25,00 & 34,10 & 60,00 & 28,20 & 96,47 & 27,00 & 64,00 & 321,09 & 33,07 & 67,46 & 103,38 & 44,63 & 25,00 & 15,18 & 49,57 \\
\hline Pres. kPa & 100 & 100 & 400 & 20 & 30 & 100 & 100 & 30 & 20 & 100 & 30 & 20 & 100 & 400 & 20 \\
\hline Flujo Mas. kg/h & 2000,25 & 237,65 & 2456,39 & 203,00 & 2253,39 & 13,93 & 2030,02 & \begin{tabular}{|l|}
2009,01 \\
\end{tabular} & 11,82 & 291,58 & 231,82 & 59,76 & 217,66 & 15,75 & 55,80 \\
\hline Frac.Mas.(Biodiesel) & 0,000 & 0,000 & 0,817 & 0,000 & 0,890 & 0,000 & 0,988 & 0,998 & 0,001 & 0,002 & 0,000 & 0,009 & 0,000 & 0,000 & 0,010 \\
\hline Frac.Mas.(Ac.Soja) & 1,000 & 0,000 & 0,001 & 0,000 & 0,001 & 0,000 & 0,002 & 002 & 0,000 & 0,000 & 0,000 & 0,000 & 0,000 & 0,000 & 0,000 \\
\hline Frac.Mas.(Glicerina) & & 0,000 & 085 & 0,000 & 93 & 0.000 & 000 & 0,000 & 0,000 & 0,716 & $\overline{00}$ & 0,000 & 0,000 & 0,000 & 0,000 \\
\hline Frac.Mas.(Metanol) & 0,000 & 0,916 & 0,089 & 1,000 & 0,007 & 0,000 & 0,004 & 0,000 & 0,750 & 0,049 & 0,000 & 0,239 & 1,000 & 1,000 & 0,133 \\
\hline \begin{tabular}{|l|} 
Frac.Mas.(Agua) \\
\end{tabular} & 0,000 & 0,000 & 0,000 & 0,000 & 000 & 1,000 & 0,001 & 0,000 & 0,249 & 0,233 & 0,100 & 0,752 & 0,000 & 0,000 & 0,857 \\
\hline \begin{tabular}{|l} 
Frac.Mas. $(\mathrm{NaOH})$ \\
\end{tabular} & 0,000 & 0,084 & 0,008 & 0,000 & 0,009 & 0,000 & 0,005 & 0,000 & 0,000 & 0,000 & 0,000 & 0,000 & 0,000 & 0,000 & 0,000 \\
\hline
\end{tabular}

\begin{tabular}{l|c|c|c|c|c|c|c|c|c|c|}
\hline \\
b) Energías
\end{tabular}

\section{\begin{tabular}{|l|l|}
\hline Energía total & $4,04 \mathrm{E}+06$ \\
\hline
\end{tabular}}




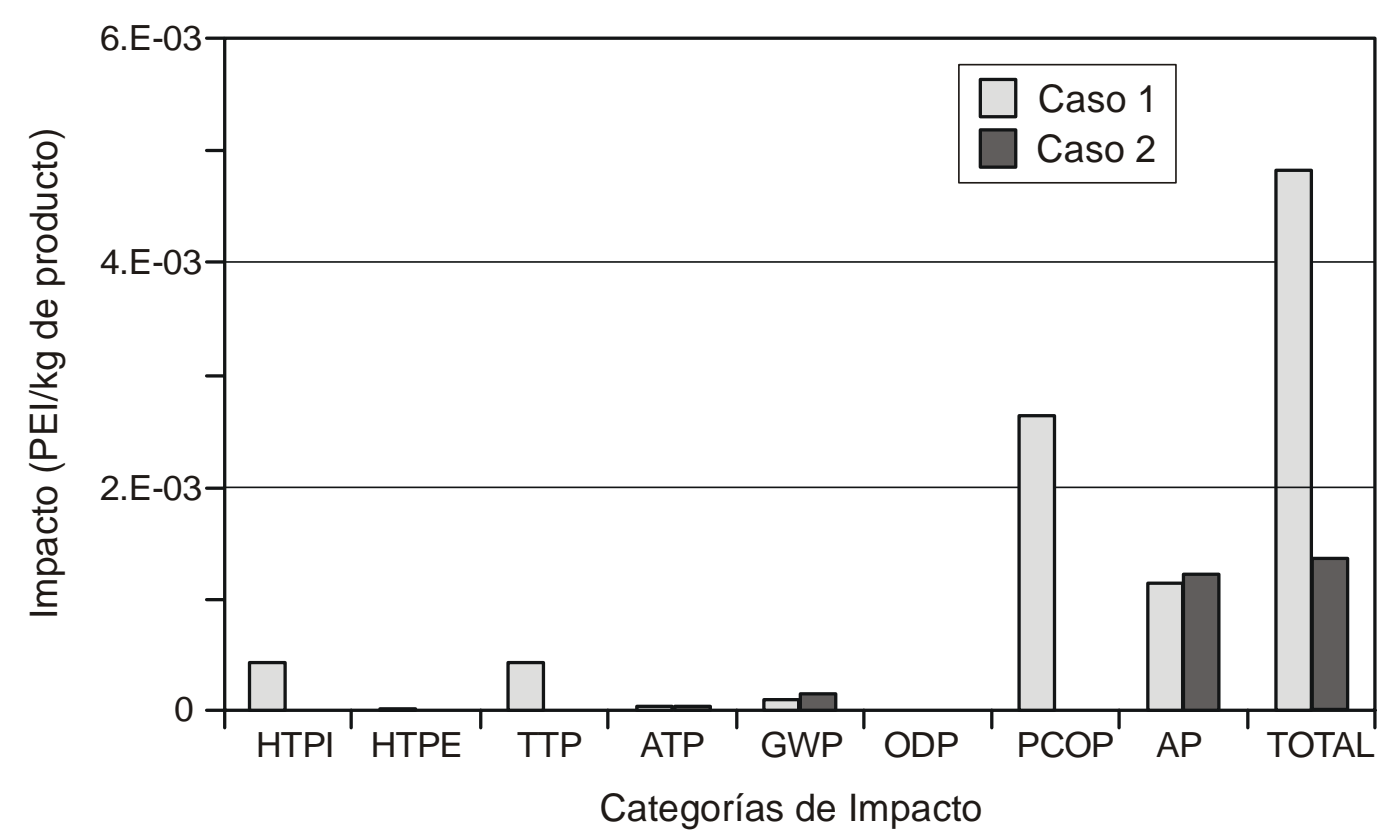

Fig. 4: Resultados del análisis de impacto ambiental para los procesos de producción de biodiesel. Caso 1 y caso 2

\section{CONCLUSIONES}

El caso 1, al consumir menos energía que el caso 2 y por ende generar menores emisiones de gases de combustión, presenta menor impacto ambiental en las categorías de GWP y AP. En el caso 2, al eliminar los efluentes que contenían metanol, se anulan todos los valores de toxicidad además del de formación de smog fotoquímico. La magnitud de los impactos HTPI, TTP y PCOP anulados en el caso 2 es mucho mayor que el incremento en GWP y AP, por lo que el índice total favorece al caso 2. Al comparar con LCA de la literatura, la recuperación de metanol dentro del proceso convencional es mucho más recomendable como proceso verde porque la producción de metanol es generadora de alto impacto ambiental.

Actualmente, las industrias muestran interés creciente en minimizar impactos desde la etapa de diseño debido al incremento en el control ambiental y a las regulaciones ambientales promulgadas. Los simuladores de procesos y el software para estimar los impactos ambientales resultan una muy buena herramienta para emprender este tipo de tareas.

\section{AGRADECIMIENTOS}

Este trabajo ha sido apoyado económicamente por el CONICET y por la Universidad Nacional del Litoral.

\section{REFERENCIAS}

Andreatta, A.E., y otros cuatro autores; Phase Equilibria in Ternary Mixtures of Methyl Oleate, Glycerol, and Methanol, Ind. Eng. Chem. Res.: 47 (15), 5157-5164 (2008).

Burgess, A.A. y D.J. Brennan; Application of Life Cycle Assessment to Chemical Processes, Chem. Eng. Science: 56 (8), 2589-2604 (2001).

Cardona, C.A., V.F. Marulanda y D. Young; Analysis of the Environmental Impact of Butylacetate Process through the WAR Algorithm, Chem. Eng. Science: 59 (24), 5839-5845 (2004).

Chiu, C.-W., M.J. Goff y G.J. Suppes; Distribution of Methanol and Catalysts between Biodiesel and Glycerin Phases, AIChE J.: 51 (4), 1274-1278 (2005).

Gerpen, J.V.; Biodiesel Processing and Production, Fuel Processing Technology: 86 (10), 1097-1107 (2005). 
Haas, M.J., A.J. McAloon, W.C. Yee y T.A. Foglia; A Process Model to Estimate Biodiesel Production Costs, Bioresource Technology: 97 (4), 671-678 (2006).

Harding, K.G., J.S. Dennis, H. von Blottnitz y S.T.L. Harrison; A Life-Cycle Comparison Between Inorganic and Biological Catalysis for the Production of Biodiesel, Journal of Cleaner Production: 16 (13), 1368-1378 (2008).

Jordan, V. y B. Gutsche; Development of an Environmentally Benign Process for the Production of Fatty Acid Methyl Esters, Chemosphere: 43 (1), 99-105 (2001).

Karaosmanoğlu, F., K.B. Ciğizoğlu, M. Tüter y S. Ertekin; Investigation of the Refining Step of Biodiesel Production, Energy and Fuels: 10 (4), 890-895 (1996).

Kiwjaroun, Ch., Ch. Tubtimdee y P. Piumsomboon; LCA Studies Comparing Biodiesel Synthesized by Conventional and Supercritical Methanol Methods, Journal of Cleaner Production: 17 (2), 143-153 (2009).

Mandagarán, B.A. y E.A. Campanella; Equilibrio de Fases en Sistemas Complejos. Casos con Aceites Vegetales, XXVII Congreso Argentino de Química, Tucumán-Argentina, Poster № 4-100, 17 al 19 de Setiembre (2008).

Negi, D.S., y otros cuatro autores; Liquid-Liquid Phase Equilibrium in Glycerol-Methanol-Methyl Oleate and Glycerol-Monoolein-Methyl Oleate Ternary System, Ind. Eng. Chem. Res.: 45 (10), 36933696 (2006).

Ranganathan, S.V., S.L. Narasimhan y K. Muthukumar; An Overview of Enzymatic Production of Biodiesel, Bioresource Technology: 99 (10), 3975-3981 (2008).

Renon, H. y J. Prausnitz; Local Composition in Thermodynamic Excess Functions for Liquid Mixtures, AIChE J.: 14 (1), 135-144 (1968).

SimaPro: Product Ecology Consultants Life Cycle Tools to Improve Environmental Performance, (2006). http://www.pre.nl/pre/products.htm\#software.

Sheehan, J.J, J.A. Duffield, R.B. Coulon y V.J. Camobreco; Life Cycle Assessment of Biodiesel versus Petroleum Diesel Fuel, Proceedings of the Intersociety Energy Conversion Engineering Conference: 3, 2140-2143 (1996).

Vasudevan, P.T. y M. Briggs; Biodiesel Production - Current State of the Art and Challenges, Journal of Industrial Microbiology and Biotechnology: 35 (5), 421-430 (2008).

Young, D.M. y H. Cabezas; Designing Sustainable Processes with Simulation: the Waste Reduction (WAR) Algorithm, Computers and Chemical Engineering: 23 (10), 1477-1491 (1999).

Young, D., R. Scharp y H. Cabezas; The Waste Reduction (WAR) Algorithm: Environmental Impacts, Energy Consumption, and Engineering Economics, Waste Management: 20 (8), 605-615 (2000).

Zhang, Y., M.A. Dubé, D.D. McLean y M. Kates; Biodiesel Production from Waste Cooking Oil: 1. Process Design and Technological Assessment, Bioresource Technology: 89 (1), 1-16 (2003 a).

Zhang, Y., M.A. Dubé, D.D. McLean y M. Kates; Biodiesel Production from Waste Cooking Oil: 2. Economic Assessment and Sensitivity Analysis, Bioresource Technology: 90 (3), 229-240 (2003b).

Zhou, W. y D.G.B. Boocock; Phase Distributions of Alcohol, Glycerol, and Catalyst in the Transesterification of Soybean Oil, JAOCS: 83 (12), 1047-1052 (2006). 